\title{
Impacto familiar nas diferentes fases da leucemia infantil'
}

\author{
Claudiane Aparecida Guimarães ${ }^{2}$ \\ Universidade de Uberaba, Uberaba - MG - Brasil \\ Sônia Regina Fiorim Enumo \\ Pontifícia Universidade Católica de Campinas, Campinas - SP - Brasil
}

\begin{abstract}
Resumo: O câncer infantojuvenil é uma doença que causa desequilíbrio físico, emocional e social em toda a família, especialmente na mãe, que é uma fonte importante de cuidado no processo da doença. Este estudo analisou o impacto familiar da leucemia em quatro estágios da doença: após o diagnóstico (mãe I), durante o tratamento (mãe 2), manutenção (mãe 3) e cuidados paliativos (mãe 4). Em aplicações individuais, as mães responderam ao PedsQL ${ }^{\mathrm{TM}}$ Family Impact Module, com 36 itens e oito dimensões. Todas as famílias foram afetadas pela doença, diferencialmente em cada fase e dimensão, em ordem decrescente: M4 (cuidados paliativos) - M2 (tratamento) - MI (diagnóstico) - M3 (manutenção); com maior impacto em atividades diárias (M2) e funcionamento cognitivo e relacionamento (M4). Avaliar esses casos clínicos, considerando o estágio da doença e variáveis emocionais maternas, pode subsidiar intervenções no processo de enfrentamento de um dos maiores estressores da vida, que é a ameaça de morte do filho.
\end{abstract}

Palavras-chave: qualidade de vida; cuidadores; câncer em crianças; estresse; crise da família.

\section{FAMILY IMPACT IN DIFFERENT STAGES OF CHILD LEUKEMIA}

\begin{abstract}
Child cancer is a disease that brings physical, emotional, and social imbalance to the patient's whole family, especially his/her mother. During the disease process, she is a unity of care and support. This study analyzed the impact of leukemia on family at four different stages. It does so based on accounts of four mothers: account of one mother related to post diagnosis (MI); one related to treatment process $(\mathrm{M} 2)$; one related to health maintenance (M3); and one related to palliative care (M4). They answered individually to Peds $\mathrm{QL}^{\mathrm{TM}}$ Family Impact Module questionnaire, which comprises 36 questions and eight dimensions. Leukemia affected all four families differently at each stage and dimension and in descending order: M4; M2; MI; M3. Disease's impact was stronger on daily activities (M2) and cognitive functioning and relationships (M4). Assessing such clinical cases by taking into account disease's stages and mother's emotional variables may help to interfere with the process of coping with a major life stressor: the threat of her child's death.
\end{abstract}

Keywords: quality of life; caregivers; cancer in children; stress; family crisis.

1 Trabalho derivado da tese de doutorado da primeira autora, orientada pela segunda (cf. Guimarães, 2015). Agradecemos à Coordenação de Aperfeiçoamento de Pessoal de Nível Superior (Capes) e ao Conselho Nacional de Desenvolvimento Científico e Tecnológico (CNPq) as bolsas de estudo e pesquisa, à psicóloga Polyana Silva Alves, à instituição e às mães participantes da pesquisa.

${ }^{2}$ Endereço para correspondência: Claudiane Aparecida Guimarães, Avenida Afonso Pena, 3.029, Bairro Brasil, Uberlândia - MG - Brasil. CEP: 38400-710.E-mail: claudianeguimaraes@yahoo.com.br. 


\begin{abstract}
Resumen: El cáncer infantil es una enfermedad que afecta física, emocional y socialmente toda la familia, especialmente la madre, que es una fuente importante de cuidados en el proceso de la enfermedad. Este estudio examinó el impacto de la leucemia en la familia basado en la experiencia de cuatro madres: la experiencia de una madre después del diagnóstico (MI); de otra durante el tratamiento (M2); de otra en mantenimiento de la salud (M3); y finalmente de una madre en materia de los cuidados paliativos. Las madres respondieron el cuestionario individual PedsQL ${ }^{\mathrm{TM}}$ Family Impact Module, con 36 itens y ocho dimensiones. Todas las familias fueran afectadas por la enfermedad de manera diferente en cada etapa y dimensión, en orden descendiente: M4; M2; MI; M3; el mayor impacto fue en las actividades diarias (M2), las relaciones personales y en el funcionamiento cognitivo (M4). Evaluar estos casos clínicos de acuerdo con las etapas de la enfermedad y las variables emocionales maternas pueden apoyar las intervenciones en el proceso de hacer frente a uno de los mayores factores de estrés de la vida: la amenaza de la muerte de un niño.
\end{abstract}

Palabras clave: calidad de vida; cuidadores; cáncer en el niño; estrés; crisis de familia.

O câncer infantojuvenil é menos frequente quando se compara sua ocorrência em adultos, equivalendo a 2,5\% dos casos, segundo o Instituto Nacional do Câncer - Inca (2014). Contudo, para essa faixa etária de zero a 19 anos, em nosso país, o câncer é a segunda causa de morte (Inca, 2014). A forma mais comum é a leucemia, com 29,9\% de incidência (Inca, 2015). Há décadas, sua incidência é estável, mas houve uma diminuição na taxa de mortalidade, com taxas de cura ou sobrevida de 70\% (Inca, 2014). Essa mudança está associada a progressos no diagnóstico e na terapêutica do câncer infantil e a modificações no acesso ao tratamento (Inca, 2014). Apesar desses indicadores, há casos em que o organismo da criança não reage positivamente ao tratamento, tem recaídas ou seu estado agravado. Essas situações impactam também os familiares em suas condições emocionais, físicas e sociais.

Conforme o estágio do câncer e a compreensão da doença, as reações emocionais da família podem ser diversas, alternando-se períodos de otimismo e esperança com períodos de desestruturação, impotência e percepção de ameaça de perda. Passado o choque inicial do diagnóstico, as preocupações se centram no estágio da doença e no tratamento, mas este último também provoca dor, fadiga e sofrimento emocional (Alves, Guirardello, \& Kurashima, 2013; Martins, Silva, \& Pires, 2011; Pöder, Ljungman, \& Essen, 2010; Sales, Matos, Mendonça, \& Marcon, 2010). Mesmo quando há prognóstico de cura, a preocupação com o filho se mantém (Alves et al., 2013). Kohlsdorf e Costa (2012, p. 120) assim sintetizam os dados de pesquisas sobre as dificuldades dos pais do paciente: "medo de recaídas, ansiedade, administração de informações recebidas, cuidados com os filhos saudáveis, tentativas de reaproximar-se de uma nova condição, cuidados com efeitos colaterais, atenção a intercorrências, entre outras, que prejudicam a qualidade de vida da família".

Esse quadro familiar tende a se complicar com o desgaste e a sobrecarga física, emocional e social, que impactam mais o cuidador principal, no caso a mãe. Nesse contexto, as pessoas podem apresentar níveis elevados de sintomas: 1. físicos - dores musculares e de cabeça, insônia, alteração no apetite e no sono, baixa resistência 
orgânica, cansaço e outros sintomas de estresse; 2. emocionais - alteração de humor, nervosismo, medo, tristeza, ansiedade, depressão, choro e percepção de sobrecarga; e 3. comportamentais - alteração de papéis e rotinas nas relações interpessoais, com restrições das atividades laborais e sociais, que podem gerar tanto aproximação quanto distanciamento, isolamento social, discussões, sentimento de culpa, de abandono e de injustiça (Alves et al., 2013; Almico \& Faro, 2014; Guimarães \& Lipp, 2011; Nóbrega \& Pereira, 2011). Essas condições incluem sintomas de transtorno de estresse pós-traumático em nível severo para $21 \%$ a $44 \%$ dos casos, podendo permanecer anos após o final do tratamento (Ljungman et al., 2014).

As estratégias de enfrentamento precisam mudar ao longo do processo de adoecimento e tratamento, pois, com o avanço da doença, ampliam-se as demandas por cuidados e a necessidade de gerenciar as próprias respostas emocionais, do paciente e dos familiares, adaptar as rotinas familiares, estabelecer comunicação satisfatória (Espírito Santo, Gaíva, Espinosa, Barbosa, \& Belasco, 2011; Kohlsdorf \& Costa, 2012). Assim, o câncer exige adaptações dos membros da família em função não só do familiar doente, mas também do cuidador familiar. Nesse processo de enfrentamento da doença, os estudos mostram que, mesmo com esse impacto negativo inicial, as famílias mantêm o otimismo quanto ao futuro do paciente e que a rotina familiar e doméstica tende a se normalizar até o final do primeiro ano após diagnóstico (Kohlsdorf \& Costa, 2012; Martins et al., 2011; Ljungman et al., 2014).

Há fatores moderadores desse impacto da doença que influenciam a vulnerabilidade do cuidador, como o tipo de suporte social, em termos de ajuda instrumental e apoio, a oferta de informações e o tempo de cuidado dedicado ao filho (Espírito Santo et al., 2011; Kohlsdorf \& Costa, 2012; Volpato \& Santos, 2007). Nesse balanço, essas famílias apresentam um nível médio de funcionamento em termos de satisfação com os relacionamentos, crescimento pessoal e manutenção do sistema familiar (Ljungman et al., 2014). Além disso, quase um terço dos pais observou um efeito positivo geral no relacionamento familiar, em termos de maior proximidade, viver mais o presente, com menos preocupações com coisas materiais (Engvall, Cernvall, Larsson, Essen, \& Mattsson, 2011; Ljungman et al., 2014). Essa maior proximidade familiar está associada com menos sintomas de depressão do que em famílias que vivem em conflito (Ljungman et al., 2014).

Diante dessa diversidade de reações dos pais, mostrando divergências nos estudos sobre o impacto da doença na família, este estudo analisou como a condição de ter um filho com leucemia afeta o cuidador familiar em diferentes fases da doença, visando obter dados comparativos e sistematizados sobre as principais áreas de impacto. Considerando que medidas específicas sobre a qualidade de vida de famílias em condições de riscos por problemas crônicos de saúde são menos comuns (Varni, Sherman, Burwinkle, Dickinson, \& Dixon, 2004), este estudo utilizou um instrumento que permite avaliar como a doença altera a qualidade de vida relacionada à saúde do cuidador em termos físicos, emocionais, sociais e cognitivos, e o funcionamento familiar, em relação aos relacionamentos e às atividades diárias. 


\section{Método}

\section{Participantes}

A amostra de conveniência foi composta por quatro mães (nomes de flores) de crianças com leucemia (nomes de jogadores de futebol), em quatro fases distintas da doença, segundo o tempo desta - entre quatro meses, dois, quatro e seis anos. Lembramos que necessariamente a doença não tem essa sequência de fases, uma vez que mais de 70\% dos casos tendem à remissão (Inca, 2014):

- Fase inicial: quatro meses após o diagnóstico, com tratamento medicamentoso para Júnior (C1), com 8 anos de idade: M1 - Dália, com 41 anos, ensino superior, casada.

- Fase de tratamento: na fila de transplante medular, com diagnóstico há dois anos para Sócrates (C2), 13 anos: M2 - Hortênsia, com 28 anos, ensino médio, divorciada, morava com a mãe.

- Fase de manutenção: após tratamento medicamentoso, com diagnóstico há quatro anos e quatro meses para Jairzinho (C3), 11 anos: M3 - Magnólia, com 49 anos, ensino médio, casada, mãe de outros dois filhos.

- Fase de cuidados paliativos: fora de possibilidades terapêuticas de cura para Zico (C4), 7 anos, com diagnóstico feito há seis anos e um mês: M4 - Kattleya, 32 anos, ensino médio, casada, mãe de outro filho.

Assim, essas mães tinham entre 32 e 49 anos de idade ( $M e=36,5$ anos); a maioria tinha o ensino médio completo e era casada; elas tinham de um a três filhos, com idade variando de 2 a 33 anos. Todas cuidavam do filho doente em tempo integral e não exerciam atividades profissionais. As crianças tinham entre 7 e 13 anos ( $\mathrm{Me}=9,5$ anos) e estavam matriculadas em escolas: três no ensino fundamental e uma no ensino médio; entretanto, três delas (C1, C2, C4) estavam impossibilitadas de frequentar aulas por causa do tratamento, que exigia internação hospitalar. Todas as crianças foram diagnosticadas com leucemia linfoblástica aguda (LLA). Eram atendidas por uma casa de apoio sem fins lucrativos de uma cidade do interior de Minas Gerais, cujo objetivo era oferecer suporte às crianças e aos adolescentes com doenças onco-hematológicas e outras hemopatias, e às suas famílias.

Os critérios de inclusão foram: ser o cuidador um familiar, não exercer o cuidado de forma remunerada e concordar em participar da pesquisa espontaneamente. Os critérios de exclusão foram: cuidador ter menos de 18 anos de idade, exceto se fosse a mãe da criança, e não responder a todos os instrumentos psicológicos da pesquisa.

\section{Instrumento}

Foi usado um instrumento específico para avaliar a qualidade de vida da família - o Pediatric Quality of Life - PedsQL ${ }^{\mathrm{TM}}$ Family Impact Module - PedsQL ${ }^{\mathrm{TM}}$-FIM (Varni et al., 2004), na versão traduzida e adaptada para o português feita por Scarpelli et al. (2008). 
Esse instrumento multidimensional é aplicado aos pais e mede o impacto da doença na qualidade de vida relacionada à saúde, nos relacionamentos e nas atividades familiares. Tem 36 itens, compondo oito dimensões/subescalas, que se referem ao funcionamento da pessoa no último mês: 1. físico (seis itens); 2. emocional (cinco itens); 3. social (quatro itens); 4. cognitivo (cinco itens); 5. comunicação (três itens); 6. preocupação (cinco itens); as outras duas dimensões abrangem dificuldades nas atividades diárias (três itens) e relações familiares (cinco itens). Há cinco opções de resposta em escala do tipo Likert ( $0=$ nunca, $1=$ quase nunca, $2=$ às vezes, $3=$ muitas vezes e 4 = quase sempre). A versão em português foi validada em amostra de Belo Horizonte, MG, com 95 pais de crianças com câncer (2-18 anos de idade), apresentando um índice geral de confiabilidade alto $(\alpha=0,89$; variação das escalas: $\alpha=0,81$ a $0,96)$, índices considerados bons (Scarpelli et al., 2008). Os escores são transformados em uma escala de 0 a 100, sendo $0=100 ; 1=75 ; 2=50 ; 3=25 ; 4=0$. É calculada a pontuação total pela soma dos escores dos 36 itens dividida pelo número de itens respondidos; calcula-se também a média dos pontos para cada uma das seis subescalas. Podem ser obtidos outros dois escores: 1. Síntese dos Escores da Qualidade de vida relacionada à saúde dos pais ou cuidadores (Parent Health-Related Quality of Life Summary Score; $\alpha=0,83$ ), escore obtido pela soma dos 20 itens dos funcionamentos físico, emocional, social e cognitivo, dividida pelo número de itens respondidos nessas subescalas; 2. Síntese dos Escores do Funcionamento Familiar (Family Summary Score; $\alpha=0,73$ ) - escore obtido pela soma dos resultados dos oito itens de Atividades Diárias e Relacionamento Familiar, dividida pelo número de itens respondidos nessas subescalas. $O$ escore total e a média do escore em cada subescala podem ser apresentados por indivíduo, como neste estudo, e calcular a média (M) e o desvio padrão (DP) do grupo, quando o $\mathrm{N}$ for maior. Com amostras pequenas, podem-se usar a mediana (Me) e o erro padrão da média (SE) para obter uma distribuição da amostra. Pelas normas, não há um ponto de corte; quanto maiores forem a pontuação total e a média, melhor será o funcionamento da família, com menor impacto negativo da doença, e vice-versa.

Além desse resultado quantitativo, durante a coleta de dados, as mães completaram os itens investigados fornecendo informações qualitativas e específicas para cada caso apresentado, o que possibilitou maior detalhamento dos dados. Esses relatos foram considerados na análise dos dados.

\section{Procedimento}

A coleta de dados foi realizada em janeiro de 2014, na instituição (M3-Magnólia), no Hospital das Clínicas de uma universidade pública (M1-Dália, M2-Hortência e M4-Kattleya) e na residência (M1-Dália e M4-Kattleya), após aprovação do Comitê de Ética em Pesquisa da instituição de ensino superior, com submissão na Plataforma Brasil (n. 548.116). O instrumento foi aplicado individualmente pela pesquisadora, psicóloga formada há sete anos, com especialização em Terapia Cognitiva-Comportamental e experiência clínica de dois anos. O tempo médio de coleta de dados com 
cada mãe foi de aproximadamente 40 minutos, sendo as sessões gravadas em áudio após autorização. Contou-se com o auxílio da psicóloga da instituição para atendimento da criança, durante a coleta de dados com as mães.

\section{Resultados}

As mães da amostra estavam lidando com a doença do filho em tempos diferentes - M1: 4 meses; M2: 2 anos; M3: 4 anos e 4 meses; e M4: 6 anos e 1 mês. Cada uma das quatro famílias estudadas apresentou um grau de impacto diferente, segundo a escala PedsQL ${ }^{T M}$-FIM: o menor resultado ocorreu na família em fase de cuidados paliativos (M4-Kattleya) ( $M=16,67)$, e o melhor funcionamento foi apresentado pela família cuja criança estava em fase de manutenção (M3-Magnólia) ( $M=81,25)$. Em ordem decrescente de impacto total da doença, tem-se a sequência, em termos de médias de pontos na escala: M4 (cuidados paliativos) - M2 (tratamento/transplante) - M1 (diagnóstico) - M3 (manutenção); no entanto, notam-se escores próximos para M1 (diagnóstico) e M2 (tratamento/transplante). Por ser uma amostra pequena, calculou-se a mediana do impacto total do grupo (Me = 55,56; $\mathrm{SE}=13,37$ ) (Tabela 1).

Tabela I. Impacto familiar da leucemia infantil nas subescalas da (PedsQL ${ }^{\mathrm{TM}}$ ) Family Impact Module ( $\mathbf{N}=4)$

\begin{tabular}{|c|c|c|c|c|c|c|}
\hline \multirow[b]{2}{*}{ PedsQLTM-FIM } & \multicolumn{6}{|c|}{ Mães } \\
\hline & $\begin{array}{c}\text { MI- } \\
\text { Dália } \\
\text { (diagnóstico) }\end{array}$ & $\begin{array}{c}\text { M2- } \\
\text { Hortência } \\
\text { (tratamento/ } \\
\text { transplante) }\end{array}$ & $\begin{array}{c}\text { M3- } \\
\text { Magnólia } \\
\text { (manutenção) }\end{array}$ & $\begin{array}{c}\text { M4- } \\
\text { Kattleya } \\
\text { (cuidados } \\
\text { paliativos) }\end{array}$ & $\begin{array}{l}\text { Mediana } \\
\text { das médias } \\
\quad(\mathrm{SE})\end{array}$ & Média (DP) \\
\hline Escore bruto & 2.100 & 1.900 & 2.925 & 600 & $\begin{array}{c}2.000 \\
(481,25)\end{array}$ & $\begin{array}{l}1.881,25 \\
(962,50)\end{array}$ \\
\hline $\begin{array}{l}\text { Impacto total } \\
\text { (Média de } 36 \text { itens) }\end{array}$ & 58,34 & 52,78 & 81,25 & 16,67 & $\begin{array}{l}\mathbf{5 5 , 5 6} \\
(13,37)\end{array}$ & $\begin{array}{c}\mathbf{5 2 , 2 6} \\
( \pm 26,74)\end{array}$ \\
\hline $\begin{array}{l}\text { Qualidade de vida } \\
\text { relacionada à saúde } \\
\text { (média de } 20 \text { itens) }\end{array}$ & 56,25 & 53,75 & 92,50 & 16,25 & $\begin{array}{c}\mathbf{5 5 , 0 0} \\
(15,57)\end{array}$ & $\begin{array}{c}\mathbf{5 4 , 6 9} \\
( \pm 31,15)\end{array}$ \\
\hline Físico (6 itens) & 29,17 & 29,17 & 100,00 & 12,50 & $\begin{array}{c}29,17 \\
(19,49)\end{array}$ & $\begin{array}{c}42,71 \\
( \pm 38,99)\end{array}$ \\
\hline Emocional (5 itens) & 65,00 & 80,00 & 100,00 & 20,00 & $\begin{array}{c}72,50 \\
(17,00)\end{array}$ & $\begin{array}{l}66,25 \\
( \pm 34)\end{array}$ \\
\hline Social (4 itens) & 87,50 & 50,00 & 68,75 & 25,00 & $\begin{array}{c}59,38 \\
(13,35)\end{array}$ & $\begin{array}{c}57,81 \\
( \pm 26,70)\end{array}$ \\
\hline Cognitivo (5 itens) & 55,00 & 60,00 & 95,00 & 10,00 & $\begin{array}{c}57,50 \\
(17,44)\end{array}$ & $\begin{array}{c}55,00 \\
( \pm 34,88)\end{array}$ \\
\hline
\end{tabular}


Tabela I. Impacto familiar da leucemia infantil nas subescalas da (PedsQL ${ }^{\mathrm{TM}}$ ) Family Impact Module ( $\mathbf{N}=\mathbf{4}$ ) (conclusão)

\begin{tabular}{|c|c|c|c|c|c|c|}
\hline \multirow[b]{2}{*}{ PedsQLTM-FIM } & \multicolumn{6}{|c|}{ Mães } \\
\hline & $\begin{array}{c}\text { MI- } \\
\text { Dália } \\
\text { (diagnóstico) }\end{array}$ & $\begin{array}{c}\text { M2- } \\
\text { Hortência } \\
\text { (tratamento/ } \\
\text { transplante) }\end{array}$ & $\begin{array}{c}\text { M3- } \\
\text { Magnólia } \\
\text { (manutenção) }\end{array}$ & $\begin{array}{c}\text { M4- } \\
\text { Kattleya } \\
\text { (cuidados } \\
\text { paliativos) }\end{array}$ & $\begin{array}{l}\text { Mediana } \\
\text { das médias } \\
\text { (SE) }\end{array}$ & Média (DP) \\
\hline $\begin{array}{l}\text { Comunicação } \\
\text { (3 itens) }\end{array}$ & 41,67 & 58,34 & 83,34 & 0,00 & $\begin{array}{c}50,01 \\
(17,5 I)\end{array}$ & $\begin{array}{c}45,84 \\
( \pm 35,03)\end{array}$ \\
\hline $\begin{array}{l}\text { Preocupação } \\
\text { (5 itens) }\end{array}$ & 30,00 & 25,00 & 60,00 & 20,00 & $\begin{array}{l}27,50 \\
(8,98)\end{array}$ & $\begin{array}{c}33,75 \\
( \pm 17,97)\end{array}$ \\
\hline $\begin{array}{l}\text { Funcionamento } \\
\text { familiar (média } \\
\text { de } 8 \text { itens) }\end{array}$ & 87,50 & 65,63 & 65,63 & 21,87 & $\begin{array}{l}65,63 \\
(13,76)\end{array}$ & $\begin{array}{c}60,16 \\
( \pm 27,53)\end{array}$ \\
\hline $\begin{array}{l}\text { Atividades diárias } \\
\text { ( } 5 \text { itens) }\end{array}$ & 75,00 & 8,34 & 83,34 & 41,67 & $\begin{array}{l}58,34 \\
(17,13)\end{array}$ & $\begin{array}{c}52,09 \\
( \pm 34,27)\end{array}$ \\
\hline $\begin{array}{l}\text { Relacionamento } \\
\text { ( } 3 \text { itens) }\end{array}$ & 95,00 & 100,00 & 55,00 & 10,00 & $\begin{array}{c}75,00 \\
(20,91)\end{array}$ & $\begin{array}{c}65,00 \\
( \pm 41,83)\end{array}$ \\
\hline
\end{tabular}

Nota: Quanto menor a pontuação, maior o impacto da doença, e vice-versa; SE (standard error) = erro padrão da média em função do tamanho da amostra; DP = desvio padrão.

Fonte: Elaborada pelas autoras.

Esse padrão dos dados se manteve na "qualidade de vida relacionada à saúde", medida pelo funcionamento físico, emocional, social e cognitivo (Me $=55,00)$. Contudo, a mediana do grupo em relação ao "funcionamento familiar" foi maior ( $\mathrm{Me}=65,63$ ), com mudança nesta sequência: M1-Dália (diagnóstico) agora apresentou um melhor

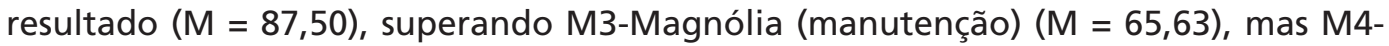
-Kattleya (cuidados paliativos) manteve-se com a menor média $(M=21,27)$ (Tabela 1).

Considerando as oito dimensões da PedsQL ${ }^{\mathrm{TM}}$-FIM, as menores médias foram obtidas por M2-Hortência (tratamento/transplante) em "atividades diárias" ( $M=8,34$ ) e M4-Kattleya (cuidados paliativos) em "relacionamento e funcionamento cognitivo" ( $M=10,00$ para ambas as dimensões). As maiores médias $(M=100)$ foram em "funcionamento físico e emocional" por M3-Magnólia (manutenção) e em "relacionamento" por M2-Hortência (tratamento/transplante) (Tabela 1).

Para toda a amostra, em ordem decrescente de impacto da doença, têm-se as seguintes dimensões, usando a mediana, dado o pequeno tamanho do grupo:

1. "Preocupação" (Me $=27,5)$, com item como "Eu me preocupo se o tratamento médico do meu filho(a) está funcionando".

2. "Funcionamento físico" (Me $=29,17)$; exemplo de item: "Eu me sinto cansado(a) quando acordo de manhã". 
3. "Funcionamento social" ( $M e=39,37)$, conforme o item "Eu me sinto isolado(a) das outras pessoas".

4. "Comunicação" (Me $=50,00)$, com item como: "Para mim, é difícil falar sobre a condição de saúde do meu filho(a) com os outros".

5. "Funcionamento cognitivo" ( $\mathrm{Me}=52,50)$, como item: "Para mim, é difícil prestar atenção nas coisas".

6. "Funcionamento emocional" ( $\mathrm{Me}=52,50)$, item exemplificado como: "Eu me sinto ansioso(a)".

7. "Atividades diárias" (Me $=58,33)$; exemplo de item: "Está difícil encontrar um tempo para terminar de fazer as tarefas da casa".

8. "Relacionamento" ( $\mathrm{Me}=75,00$ ), exemplo: "Existe falta de comunicação entre as pessoas da sua família" (Tabela 1).

As preocupações dessas mães centravam-se em lidar com os sintomas físicos decorrentes dessa situação nas alterações na rotina diária de todos envolvidos no processo da doença, exigindo mudança de papéis e adaptações e nas mudanças nos relacionamentos, envolvendo outros membros da família, amigos e parentes.

Lidar com os próprios sintomas físicos era uma das dificuldades dessas mães, como se vê nestes relatos:

Acordo de noite com muita dor de cabeça (M1-Dália - diagnóstico).

Eu sinto meu corpo muito pesado. Porque, quando ele está passando mal ou coisa assim, parece que as pernas ficam pesadas, o coração dispara, a respiração não vem, principalmente quando ele dá reação (M4-Kattleya - cuidados paliativos).

O processo da doença altera a rotina diária de paciente, cuidador e família, e impõe mudança de papéis e adaptações, como ilustra o relato de M2-Hortência (tratamento/ transplante): "Enquanto um tá cuidando dele, a outra tá fazendo isso... Porque é um momento que precisa de muita limpeza da casa, principalmente... é remédio toda hora, é alimento toda hora, aí vem e passa mal".

Também os relacionamentos com outros membros da família, amigos e parentes foram alterados, o que implicava afastamento do convívio social e isolamento. No caso da M3-Magnólia (manutenção), nessa fase da doença do filho, ela buscava retomar o convívio com outras pessoas a fim de inserir o filho em outros meios, como mostra este relato: "Eu não tenho essa vida social, mas a gente tá começando a engatinhar, sabe? Retomar, por exemplo, semana passada, a gente foi no clube".

Quando se analisam as respostas de cada mãe em ordem decrescente de impacto familiar e de qualidade de vida, vê-se que M4-Kattleya (cuidados paliativos) apresentou médias menores em metade das oito dimensões da PedsQL ${ }^{\mathrm{TM}}$-FIM: "comunicação" $(M=0,00)$, "funcionamento cognitivo" $(M=10,00)$, "relacionamento" $(M=10,00)$ e "funcionamento físico" $(M=12,50)$ (Tabela 1). A maior dificuldade foi na área da "comunicação", exemplificada pelo seguinte relato: "Eu escrevo na agenda, lá tem tanta 
coisa que a gente já passou. Porque chega um momento que eu não consigo conversar com as pessoas, aí eu prefiro escrever" (M4-Kattleya - cuidados paliativos).

As verbalizações deixam claro que a prioridade, no momento, era a criança e que dedicava o tempo integralmente para cuidar do filho. Isso pode explicar a maior média do grupo na subescala de "atividades diárias" ( $M=41,67)$ (Tabela 1), como se observa neste relato: "Ultimamente, eu não estou com vontade de nada, só de ficar perto dele. Às vezes, eu tenho vontade de fazer as coisas, aí começa a fazer e já desanima para mim, o importante é ele" (M4-Katlleya - cuidados paliativos).

Embora a "comunicação" seja uma das áreas de maior dificuldade para as pessoas na fase terminal de doenças, M4-Kattleya considerava que esse aspecto era uma característica dela: "Eu sempre afastei, sempre fui tratada diferente. Depois que ele ficou doente, aí a gente nem sai mais".

Além disso, por causa do tempo de tratamento e da gravidade do caso, ela preferia se isolar, para proteger o filho, para não o expor, e evitar que ele escutasse comentários sobre o avanço da doença. Decorrem daí o isolamento e a falta de comunicação, como se vê no relato de M4-Kattleya (cuidados paliativos):

Sempre foi nós dois, desde que ele foi diagnosticado. Eu recebi a notícia sozinha, eu procurei ajuda sozinha, eu fique aqui com ele um mês sem sair para nada, só eu e ele. Ele não teve visita, eu não tinha ninguém. [...] Eu prefiro não ficar tocando no assunto, principalmente para ele não se sentir diferente.

Observaram-se uma ambivalência de sentimentos entre conformismo e esperança de reversão no quadro clínico, e o reconhecimento da gravidade e da iminência da morte, como sugere o seguinte relato: "É como se eu tivesse uma bomba-relógio, com pavios bem curtos, prestes a explodir a qualquer momento" (M4-Kattleya - cuidados paliativos).

O segundo lugar em termos gerais de impacto da doença ficou com M2-Hortência (tratamento/transplante), que apresentou uma média do escore bruto de 52,78 pontos, com média menor em "atividades diárias" $(M=8,34)$ e maior em "funcionamento físico" ( $M=29,17)$ (Tabela 1). O impacto na rotina era grande, mesmo com ajuda da mãe, como mostra este relato:

É um momento que precisa muito da limpeza da casa principalmente, entendeu? Mas é remédio toda hora, é alimento toda hora, aí, vem e passa mal; você acabou de fazer uma coisa, ele não quer comer, aí você faz outra, aí não quer comer, você faz outra; então é... - pode pôr aí - quase sempre. No modo geral, a gente faz, mas minha mãe ajuda (M2-Hortência - tratamento/transplante).

O melhor resultado de M2-Hortência (tratamento/transplante) foi na dimensão "relacionamento" ( $M=100)$, coerente com o suporte familiar, o que era feito sem conflitos: "Problema nenhum, conflitos 'minimíssimos' assim. A gente se dá superbem. [...] "Ela é uma mãezona, graças a Deus eu tenho ela". 
A participante M1-Dália, que havia recebido o diagnóstico de câncer do filho fazia quatro meses, ficou em terceiro lugar $(M=58,34)$. A menor média dela foi em "funcionamento físico" $(M=29,17)$ (Tabela 1). A coleta de dados com essa mãe foi feita um dia após um período de internação do filho, o que pode justificar um pior funcionamento nessa dimensão. Ela enumerou os sintomas físicos apresentados ante a situação de cuidar do filho: "Eu não sei se é porque eu durmo mal, acordo mais vezes assim, então eu levanto um bagaço... [...] Muitas vezes, eu tenho muita dor de cabeça. Parece, assim, que meu corpo fica dolorido, sabe? Como se passou uma patrola nele".

A melhor média de M1-Dália (diagnóstico) foi na dimensão "relacionamento" $(M=95,00)$ (Tabela 1), por estar possivelmente à rede de apoio familiar, que procurava suprir as demandas da criança, que era o único neto homem. O relato a seguir ilustra essa situação: "Da minha família e do meu marido sempre, das minhas irmãs, dos meus amigos também. Nossa! Me ajudaram bastante".

Por fim, M3-Magnólia (manutenção) foi a mãe que apresentou as maiores médias $(M=81,25)$, especialmente em "funcionamento físico" $(M=100)$ e "funcionamento emocional" ( $M=100)$ (Tabela 1). Esses resultados podem ser justificados pelo fato de o tratamento do filho de M3-Magnólia estar em fase de manutenção desde 2012, com prognóstico de cura total. Assim, ela podia cuidar de si, já que o filho não demandava atenção por tempo integral. A mãe recorria à fé como forma de lidar com essa situação, como observado nestes relatos: "Eu não deixo essa esperança escapar do meu coração. Eu me encho de esperança, eu não deixo hora nenhuma me faltar esperança, esperança de que agora para frente vai ser melhor, e melhor e melhor, sabe?".

Apesar do apoio do marido, das filhas adultas e de amigos, a menor média de M3-Magnólia (manutenção) foi na dimensão "relacionamento" $(M=55,00)$ (Tabela 1). Ponderou, porém, que a família conseguia chegar a soluções positivas para o bem de todos, como se vê no relato: "Eu acho, assim, que, quando a gente se determina para um caso específico; quando a gente se une, a gente consegue resolver da melhor maneira".

\section{Discussão}

A análise dos dados dessas quatro mães possibilitou verificar que a doença tem impacto diverso em cada família que lidava com a doença em tempos diferentes. No primeiro momento, ao choque no diagnóstico da doença soma-se o impacto de cuidar do filho, gerando perturbações psicológicas, emocionais e físicas. Contudo, também ocorreram relatos de esperança na cura e na boa recuperação, e bom funcionamento familiar, confirmando dados da literatura (Ljungman et al., 2014; Martins et al., 2011). As mudanças, as dificuldades, os sentimentos e o impacto da doença puderam ser avaliados em todas as famílias, marcadamente nas dimensões físicas, nas atividades diárias, nos relacionamentos e na comunicação, à semelhança dos dados da área (Alves et al., 2013; Almico \& Faro, 2014; Guimarães \& Lipp, 2011; Kohlsdorf \& Costa, 2012; Nóbrega \& Pereira, 2011). Nesse ponto, este estudo se diferencia de outros trabalhos com desenho qualitativo que utilizaram um instrumento específico para a avaliação 
do impacto familiar e da qualidade de vida, como a escala PedsQL ${ }^{\text {TM }}$-FIM (Varni et al., 2004) adotada aqui. Por meio dessa escala, obteve-se um quadro resumidor e, ao mesmo tempo, indicativo de áreas a serem incluídas em intervenções com essa população. Além das análises individuais, podem-se obter também padrões de grupos de pais ou cuidadores no contexto de doenças crônicas. Nesse sentido, este trabalho contribui em termos metodológicos ao mostrar as possibilidades de análise para o estudo de casos de famílias com crianças em diferentes fases do câncer.

Com esse instrumento, foi possível identificar que o impacto se diferencia segundo o tempo de duração e fase da doença, sendo maior na família da criança fora de possibilidades terapêuticas de cura (M4) que lidava com o problema havia mais de seis anos e menor na família da criança em fase de manutenção (M3), com diagnóstico feito há quatro anos e quatro meses. Identificaram-se reações e comportamentos comuns às famílias, como a preocupação sobre a eficácia do tratamento do filho (M2 e M4) e o cansaço diário devido à dedicação total aos cuidados da criança (M1, M2 e M4). Condições distintas também puderam ser identificadas, como a dificuldade de comunicação expressada por M4 e menos evidentes em outras mães, e o manejo dos relacionamentos familiares, também mais impactante na família de M4, que estava cuidando do filho em fase de cuidados paliativos. Esses comportamentos ocorreram conforme a prioridade do momento e das dificuldades apresentadas pela fase da doença em que a criança se encontrava, dados que vão ao encontro aos apresentados por Espírito Santo et al. (2011) e Volpato e Santos (2007).

As exigências provenientes desse processo provocam alterações nos papéis do cuidador e de outros membros da família, e nas relações e atividades sociais, como apontado pelas mães. Entretanto, também ocorrem adaptações baseadas no suporte social e familiar, na comunicação e na esperança e fé. Nessas condições, como a família se sente motivada com sentimentos positivos, ela pode superar o sofrimento e assumir o papel de cuidadora (Martins et al., 2011).

Independentemente da fase da doença, o impacto, a sobrecarga e as dificuldades são excessivos, e cada núcleo familiar, em seu contexto e com suas experiências, recorre a estratégias de enfrentamento para lidar com a situação. Essas estratégias podem ser compreendidas como um conceito multidimensional, parte do processo de autorregulação emocional, fisiológica, cognitiva, motivacional e do comportamento. Integram as relações do indivíduo e o meio, e são descritas por atitudes, respostas, cognições e comportamentos adotados na interação. Assim, podem variar de acordo com o estressor, o domínio do indivíduo e o momento do processo de enfrentamento (Zimmer-Gembeck \& Skinner, 2011). Essas condições podem explicar as diferenças individuais no impacto da doença encontradas nessa amostra de mães.

Nesse processo de enfrentamento, o apoio familiar, por meio do acolhimento e da ajuda nos cuidados práticos com o filho, a comunicação assertiva entre os envolvidos no cuidado da criança e as tomadas de decisão conjunta entre os membros da família amenizam a sobrecarga do cuidador principal, nesse caso as mães, como ressaltado por Martins et al. (2011). Alves et al. (2013) salientam também que o apoio da equipe de profissionais, a partir do auxílio aos pais no gerenciamento do estresse e do esta- 
belecimento de uma relação de confiança, pode colaborar para minimizar as dificuldades enfrentadas pelos pais.

Os dados aqui apresentados evidenciam a importância de identificar as demandas dessa população que vivencia a experiência de cuidar de um filho com câncer. São pessoas que necessitam de uma escuta acolhedora, assim como de oportunidade para expressar sentimentos, receios, preocupações e significados desse processo. O apoio adequado da equipe de profissionais, das redes de apoio e das comunidades religiosas pode amenizar as dificuldades. Ressalta-se aqui a necessidade de um conhecimento básico e técnico para esse apoio ser eficiente. Para tanto, são necessárias a formação continuada dos profissionais da saúde e de voluntários e grupos de estudos, a elaboração de propostas de intervenção e a divulgação de informações claras sobre saúde, doença e cuidar.

Este estudo não pretendeu analisar as fases da doença, mas o impacto dela nas famílias que estavam em processos distintos, segundo a duração da doença. Apesar das limitações quanto ao tamanho da amostra, os resultados deste estudo podem contribuir para a área da Psicologia Pediátrica no país, com dados mais sistematizados sobre como o impacto da doença afeta as famílias, principalmente as mães.

\section{Referências}

Almico, T., \& Faro, A. (2014). Enfrentamento de cuidadores de crianças com câncer em processo de quimioterapia. Psicologia, Saúde \& Doenças, 15(3), 724-738. DOI: 10.15309/14psd150313.

Alves, D. F. S., Guirardello, E. B., \& Kurashima, A. Y. (2013). Estresse relacionado ao cuidado: o impacto do câncer infantil na vida dos pais. Revista Latino-Americana Enfermagem, 21(1). Recuperado em 10 outubro, 2014, de http://www.scielo.br/ scielo.php?pid=S010411692013000100010\&script=sci_arttext\&tlng=pt.

Engvall, G., Cernvall, M., Larsson, G., Essen, L., von, \& Mattsson, E. (2011). Cancer during adolescence: negative and positive consequences reported three and four years after diagnosis. PLOS ONE, 6(12), 1-5. DOI: 10.1371/journal.pone.0029001.

Espírito Santo, E. A. R., Gaíva, M. A. M., Espinosa, M. M., Barbosa, D. A., \& Belasco, A. G. S. (2011). Cuidando da criança com câncer: avaliação da sobrecarga e qualidade de vida dos cuidadores. Revista Latino-Americana de Enfermagem, 19(3). Recuperado em 20 janeiro, 2014, de http://www.eerp.usp.br/rlae.

Guimarães, C. A. (2015). Cuidadores familiares de pacientes oncológicos pediátricos em fases distintas da doença: processo de enfrentamento. Tese de doutorado, Pontifícia Universidade Católica de Campinas, Campinas, SP, Brasil.

Guimarães, C. A., \& Lipp, M. E. N. (2011). Um olhar sobre o cuidador de pacientes oncológicos recebendo cuidados paliativos. Psicologia: Teoria e Prática, 13(2), 50-62.

Instituto Nacional de Câncer "José Alencar Gomes da Silva" (2014). Diagnóstico precoce do câncer na criança e no adolescente (2a ed., rev. ampl., 3a reimp.). Rio de Janeiro: Inca. 
Instituto Nacional de Câncer "José Alencar Gomes da Silva" (2015, janeiro/julho). Perfil da assistência oncológica no Brasil, de 2007 a 2011. Informativo Vigilância do Câncer, 6.

Kohlsdorf, M., \& Costa, A. L., Júnior (2012). Impacto psicossocial do câncer pediátrico para pais: revisão da literatura. Paidéia, 22(51), 119-129. DOI: 10.1590/ S0103-863X2012000100014.

Ljungman, L., Cernvall, M., Grönqvist, H., Ljótsson, B., Ljungman, G., \& Essen, L., von (2014). Long-term positive and negative psychological late effects for parents of childhood cancer survivors: a systematic review. PloS ONE, 9(7), 1-15. DOI: 10.1371/journal.pone.0103340.

Martins, C. B. S., Silva, N., Filho, \& Pires, M. L. N. (2011). Estratégias de coping e o impacto sofrido pela família quando um dos seus está em tratamento contra o câncer. Mudanças - Psicologia da Saúde, 19(1-2), 11-18.

Nóbrega, K. I. M., \& Pereira, C. U. (2011). Qualidade de vida, ansiedade e depressão em cuidadores de crianças com neoplasia cerebral. Psicologia: Teoria e Prática, 13(1), 48-61. DOI: 10.1590/1982-370002332013.

Pöder, U., Ljungman, G., \& Essen, L., von (2010). Parents' perceptions of their children's cancer-related symptoms during treatment: a prospective, longitudinal study. Journal of Pain and Symptom Management, 40(5), 661-670. DOI: 10.1016/j. jpainsymman.2010.02.012.

Sales, C. A., Matos, P. C. B., Mendonça, D. P. R., \& Marcon, S. S. (2010, outubro/ dezembro). Cuidar de um familiar com câncer: o impacto no cotidiano de vida do cuidador. Revista Eletrônica de Enfermagem, 12(4), 616-621. Recuperado em 20 janeiro, 2014, de http://dx.doi.org/10.5216/ree.v12i4.12160.

Scarpelli, A. C., Paiva, S. M., Pordeus, I. A., Varni, J. W., Viegas, C. M., \& Allison, P. J. (2008). The Pediatric Quality of Life Inventory ${ }^{\mathrm{TM}}$ (PedsQL ${ }^{\mathrm{TM}}$ ) Family Impact Module: reliability and validity of the Brazilian version. Health and Quality of Life Outcomes, 6(35), 1-8. DOI: 10.1186/1477-7525-6-35.

Varni, J. W., Sherman, S. A., Burwinkle, T. M., Dickinson, P. E., \& Dixon, P. (2004). The PedsQL Family Impact Module: preliminary reliability and validity. Health and Quality of Life Outcomes, 2, 55- 1-6. DOI: 10.1186/1477-7525-2-55.

Volpato, F. S., \& Santos, F. R. S. (2007). Pacientes oncológicos: um olhar sobre as dificuldades vivenciadas pelos familiares cuidadores. Imaginário - USP, 13(14), 511-544.

Zimmer-Gembeck, M. J., \& Skinner, E. A. (2011). The development of coping across childhood and adolescence: an integrative review and critique of research. International Journal of Behavioral Development, 35(1), 1-17. DOI: 10.1177/01650254 10384923.

Submissão: 20.3 .2015

Aceitação: 10.8 .2015 